\title{
BMJ Open Anxiety and depression levels of the general population during the rapid progressing stage in the coronavirus disease 2019 outbreak: a cross-sectional online investigation in China
}

\author{
Zeya Shi (D) , ${ }^{1}$ Yuelan Qin, ${ }^{1}$ Sek Ying Chair, ${ }^{2}$ Yanhui Liu, ${ }^{3}$ Yu Tian, ${ }^{4}$ Xin Li, ${ }^{1}$ \\ Wanqin $\mathrm{Hu},{ }^{1}$ Qun Wang (i) ${ }^{5}$
}

To cite: Shi Z, Qin Y, Chair SY, et al. Anxiety and depression levels of the general population during the rapid progressing stage in the coronavirus disease 2019 outbreak: a crosssectional online investigation in China. BMJ Open 2021;11:e050084. doi:10.1136/ bmjopen-2021-050084

- Prepublication history and additional supplemental material for this paper are available online. To view these files, please visit the journal online (http://dx.doi.org/10.1136/ bmjopen-2021-050084).

Received 14 February 2021 Revised 08 April 2021 Accepted 29 April 2021

Check for updates

(C) Author(s) (or their employer(s)) 2021. Re-use permitted under CC BY-NC. No commercial re-use. See rights and permissions. Published by BMJ.

For numbered affiliations see end of article.

Correspondence to

Qun Wang;

qunwang@szu.edu.cn

\section{ABSTRACT}

Objective The outbreak of COVID-19 has major impacts on the psychological health of the public. This study aimed to investigate the anxiety and depression levels of the general population during the rapid progressing stage of COVID-19 pandemic in China and to explore the associated factors.

Design and setting A cross-sectional online survey. Participants 2651 Chinese people.

Measures The Hospital Anxiety and Depression Scale was used to measure their psychological health. A structured questionnaire collected possible associated factors, including sociodemographic characteristics, health information, contact history-related information, experience and perceptions, knowledge and education and adopted precautions. Multiple linear regression was conducted to explore the factors associated with anxiety and depression.

Results The mean score of anxiety and depression was 4.35 and 4.38 , respectively. The rates of people with anxiety and depressive symptoms (with $>7$ score in the subscale) were $14.15 \%$ and $17.35 \%$, respectively. Participants without political party membership, with contact history of COVID-19, going out or gathering, taking Chinese medicine herbs, being unsatisfied with current precautions, perceiving higher risks of infection, lower knowledge and poorer health presented higher anxiety and depression levels. Moreover, those who were females, married, lived alone and wore mask were more anxious; whereas people who were younger, experienced public health crisis, did not take precautions (regular work-rest, exercise) had higher depression level. Conclusions During the rapid progressing stage of COVID-19 pandemic in China, one-seventh and one-sixth respondents presented anxiety and depression symptoms, respectively. The risk factors for anxiety and depression included the following: without political party membership, with contact history of COVID-19, going out or gathering, wearing masks, taking Chinese medicine herbs, being unsatisfied with current precautions, perceiving higher susceptibility, lower knowledge and poorer health status. Extensive information and psychological support should be provided to improve the mental health of the general population.
Strengths and limitations of this study

- A nationwide online study with a large sample.

- A study was conducted when the daily new cases of COVID-19 reached the peak in China.

- Employment of validated instrument for measuring psychological health.

- Results are mostly limited to the cross-sectional design with snowball sampling.

The use of self-reported data.

\section{INTRODUCTION}

In December 2019, the COVID-19 became an epidemic in China and later, a global pandemic. This is the third outbreak of human coronavirus in the past two decades after the SARS in 2003 and the Middle East respiratory syndrome (MERS) in 2015. The novel coronavirus has strong capability of human-to-human transmission, leading to the rapid global spread. ${ }^{1}$ On 31 January 2020, the number of COVID-19 cases reached 11791 in China, and the WHO declared the COVID-19 outbreak to be a public health emergency of international concern. ${ }^{2}$ The number of cases increased rapidly since then. According to the data from the Chinese National Health Commission (NHC), the initial number of COVID-19 cases was 40 171, with 908 deaths until 9 February 2020 (before the launch of the current study). ${ }^{3}$ This figure rapidly increased to 74576 cases with 2118 deaths after 10 days. ${ }^{4}$ The peak of this pandemic in China occurred on 12 February 2020, with 15152 new cases and 254 deaths on that day. ${ }^{5}$ The global pandemic lasted for a year. By 8 February 2021, there were 105805951 confirmed cases in the world, including 2312278 deaths. ${ }^{6}$ In response to the original outbreak, China activated various emergency 
responses, such as the lockdown of Wuhan (the epicentre in China), a nationwide quarantine policy, building specialty and Fang Cang Shelter hospitals and sending tens of thousands of healthcare professionals to support Wuhan. ${ }^{7}$

The coronavirus outbreak also had major psychosocial implications. ${ }^{8}$ The rapid evolution of the COVID-19 epidemic, personal health concerns and implementation of precautionary policies (eg, quarantine orders and travel restrictions), possibly created psychological distress, including anxiety, depression and anger. Moreover, the extensive media coverage of the outbreak may trigger or aggravate global anxiety and post-traumatic stress disorder (PTSD). The outbreak of SARS increased the prevalence of depression symptoms in Taiwan $(3.7 \%){ }^{9}$ Toronto $(31.2 \%)^{10}$ and Hong Kong $(15.6 \%) .{ }^{11}$ The presence of PTSD was as high as $25.6 \%-28.9 \%$ among those who were quarantined or infected. ${ }^{10}{ }^{11}$ During MERS, $7.6 \%$ of Korean public showed symptoms of anxiety, and the prevalence remained $3.0 \%$ at $4-6$ months after the outbreak. ${ }^{12}$ To cope with the psychological impacts, the NHC published the Intervention Guidelines for Psychological Crisis during the COVID-19 pandemic (NHC Guideline) on 26 January 2020. ${ }^{13}$ The psychological responses of the general populations include panic, anxiety, disappointment, giving up, fear of going out, unreasonable disinfection, aggressive behaviours or over-optimism. ${ }^{13}$ People may have different psychological responses to different types of contagious diseases and at different stages of the outbreaks. However, little is known about the actual psychological responses of the general population at the rapid progressing stage of this COVID-19 pandemic.

Understanding the factors associated with the psychological responses of the general population can help identify the high-risk groups for psychological disorders and develop specific interventions. Some studies have reported various factors influencing psychological health during SARS and MERS. People who were female, younger aged, married, with higher education and living alone had higher anxiety and depression levels. ${ }^{10} 1415$ Additionally, with contact history of the coronavirus, perceived poor health status, greater severity and susceptibility of the disease were associated with higher anxiety and depression levels. ${ }^{9101415}$ Lack of experience in contagious disease outbreaks or knowledge about the coronavirus could trigger depression symptoms, ${ }^{10}$ whereas satisfaction with preparedness of the government is associated with better psychological health. ${ }^{16}$

Various studies reported the psychological responses in different populations at different stages of this COVID-19 pandemic. A systematic review reported a $31.9 \%$ prevalence of anxiety (95\% CI: 27.5 to 36.7 ) among general populations in the current COVID-19 pandemic, and a similar prevalence of $33.7 \%$ (95\% CI: 27.5 to 30.6) for depression. ${ }^{17}$ That study also summarised the high-risk groups of people for psychological disorders. People who were female, 21-40 years old, with higher education, with chronic illness, without accurate knowledge or information about the pandemic reported higher prevalence of anxiety or depression. Another systematic review reported a $46 \%$ prevalence of anxiety symptoms (95\% CI: 33.9 to 58.2) from 16 studies with 25755 participants in coronavirus epidemics, including SARS, MERS and COVID-19. ${ }^{18}$ This systematic review did not identify age or gender as significant moderators for anxiety symptoms. Thus, no consensus about the factors associated with anxiety or depression during the pandemic was achieved.

To date, most studies among Chinese were conducted either during the initial stage (end of January to 8 February 2020) or in the postpeak stage (13 February 2020 or after) of the pandemic. ${ }^{19-24}$ Before the peak in China, the prevalence of anxiety ranged from $12.2 \%$ to $28.8 \%$, whereas the data of depression ranged ranged from $11.0 \%$ to $26.16 \% .{ }^{19-21}$ After the peak, the prevalence of anxiety and depression symptoms revealed increasing trends $(20.8 \%$ to $44.2 \%$ for anxiety, $19.5 \%$ to $64.6 \%$ for depression). ${ }^{22-24}$ However, limited evidence is available regarding the anxiety and depression levels of the general population at the rapid progressing stage of COVID-19 in China, especially when the daily new cases reached the peak.

Therefore, the current study was conducted to identify the anxiety and depression levels of the general population during the rapid progressing stage in COVID-19 pandemic in China and to explore their associated factors. The study findings can help understand people's psychological responses in the peak stage of a pandemic and provide valuable references for developing psychological interventions to high-risk groups.

\section{METHODS}

\section{Study design and participants}

To eliminate close personal contacts and mass gatherings during the COVID-19 outbreak, ${ }^{6}$ this cross-sectional study used an online survey with snowball sampling. The online survey was distributed from 10 to 19 February 2020 in China, when the number of daily cases was rapidly increasing and reached the peak. People who received the survey link could complete the survey and send the link to others. All participants were recruited online by the research team.

\section{Study instrument}

Considering its good validity, reliability and conciseness, the Hospital Anxiety and Depression Scale (HADS) was used to measure anxiety and depression. HADS is a 14-item four-point Likert scale with the subscales for anxiety (7-item) and depression (7-item).${ }^{25}$ Each subscale ranges from 0 to 21 (0-3 for each item), and a higher score indicates a higher level of anxiety/depression. An overall score $\geq 15$ indicates psychological distress. A subscale score $\leq 7$ indicates no symptom of anxiety or depression, whereas $\geq 11$ indicates obvious symptoms. A subscale score $>7$ reveals good sensitivity and specificity 
in screening anxiety/depression, ${ }^{25}$ which was adopted in the current study. HADS has established good psychometric properties in different Chinese populations, including students, general populations and community residents. ${ }^{26-28}$ In the current study, this instrument revealed good internal consistency with a Cronbach's $\alpha$ of 0.817 for the whole scale, 0.755 for the anxiety subscale and 0.791 for the depression subscale.

A structured questionnaire was used (online supplemental file 1) to explore the possible factors related to anxiety and depression among the general population during the COVID-19 pandemic. Based on a literature review about influencing factors of anxiety or depression, ${ }^{9-16}$ the current study collected the following data: (a) sociodemographic characteristics, including age, gender, education, working status, political party membership, marital status, monthly income and living conditions; (b) perceived health status, measured by a visual analogue scale (VAS $=0$ indicating the poorest health and 100 indicating the best health); (c) contact history-related information, including travel history to Hubei in the past 2 weeks, close contact with any COVID-19 patient, whether received quarantine, with COVID-19 patient(s) in the family or the community and distance to the nearest COVID-19 patient and hospital (in kilometres); (d) experiences and perceptions, including previous experience in a similar public health crisis (with 'yes' or 'no' choices), perceived risk of being infected (VAS $=0$ indicating no risk and 100 indicating definitely being infected); (e) knowledge and education needs, including whether received education on COVID-19, self-rated level of knowledge (VAS $=0$ indicating no knowledge and 100 indicating full knowledge) and needs for further education and (f) adopted precautions, including the types of precautions adopted by the community, family and individuals; the frequency of going out; whether wore masks when going out; types of masks and satisfaction level to current precautions (six-point Likert choices).

To examine its readability, clarity and length, the study instrument was pretested among 20 people who were invited from a Wechat group of a community centre in Changsha, Hunan. No changes were required after the pretest. The questionnaire could be completed within $15 \mathrm{~min}$.

\section{Patient and public involvement}

Patients and/or the public were not involved in the design, or conduct, or reporting, or dissemination plans of this research.

\section{Data collection}

The questionnaire was presented in the Wenjuanxing online investigation platform (www.wjx.cn, Ranxing Information Technology Co.). The research team widely sent the survey link to possible participants through WeChat and QQ groups, including people in our contact list, member groups of community centres, learning clubs and working groups. Following snowball sampling, people who received the survey link were encouraged to send it to others. People could click the link and launch the first page, which introduced the aims and process of the survey. At the end of the first page, the question 'Do you agree to participate in this survey' was asked to acquire the consent of the participants. Only the 'Yes' option led to the next page for the questionnaires. The first item in the questionnaire asked about age. If the answer was under 16 , the questionnaire would be led back to the study introduction and consent form page, which required the electronic signature of his/her parent or guardian. All the consent forms were available for the researchers in the online investigation system. One mobile IP could only submit the answers only once to prevent duplication. The online survey was discontinued 10 days later.

\section{Statistical analysis}

Data were exported from the Wenjuanxing system. SPSS V.25.0 was used for data analysis. The statistics of mean, $\mathrm{SD}$, count and percentage were used to describe the variables. T-test and one-way analysis of variance were used to explore the differences in HADS among participants with various characteristics. Multiple linear regression with stepwise method was conducted to explore the factors associated with anxiety and depression. Linear correlation between the dependent variables and continuous independent variables, multicollinearity and residual analysis were carefully checked for the multiple linear regression. ${ }^{29}{ }^{30}$ Beta, SE and the $95 \%$ CIs were computed. Multicollinearity was excluded by the findings that all Pearson correlation coefficients between independent variables $<0.5$, and all tolerance values $>0.780 .{ }^{30}$ The DubinWastson values were 1.984 and 2.027 for the anxiety and depression regression model, respectively, indicating the independency of residuals. ${ }^{31}$ The histogram, P-P plots and scatter-plots revealed that the residuals met normal distribution and homogeneity of variance. The significance level was set as 0.05 .

\section{RESULTS}

A total of 2711 responses were received, including 60 without consent. Finally, 2651 responses from 217 cities of 30 provinces in China were analysed. Among the participants, $23.3 \%$ reported the presence of anxiety or depression symptoms (with $>7$ score in either subscale of HADS), including $8.2 \%$ with both symptoms.

\section{Characteristics of the respondents}

Most participants were female $(78.54 \%)$, with no political party membership $(50.74 \%)$, with a bachelor's degree or above $(83.65 \%)$, married $(72.46 \%)$, employed $(83.86 \%)$, at working $(55.87 \%)$, with $>3000$ RMB personal monthly income $(78.27 \%)$, living in urban areas $(84.31 \%)$ and living with their family $(84.61 \%)$. The mean age of the participants was 35.91 $(\mathrm{SD}=10.65)$ years. The characteristics of the participants are summarised in table 1. 
Regarding the contact information, most respondents reported no travel history to Hubei in the past 2 weeks (97.89\%), had no close contact with COVID-19 patients $(94.57 \%)$, were not quarantined $(78.86 \%)$ and with no COVID-19 patient in their family members or neighbours $(85.67 \%)$. In the past week, $67.22 \%$ of the respondents went out for less than seven times (including $33.61 \%$ less than once), and $38.82 \%$ had been at home for 3 days or longer. The majority of the respondents $(97.06 \%)$ wore masks when outside the home, and the disposable protective masks were extensively adopted $(93.10 \%)$. The adopted precautions by individuals included wearing masks (95.85\%), washing hands frequently (97.40\%) and not going out or gathering $(91.81 \%)$. In general, $90.53 \%$ of the respondents were satisfied with current precautions.

The participants reported a mean self-perceived health score of 84.13 ( $\mathrm{SD}=17.00)$. The self-perceived risk of being infected by COVID-19 was low, with a mean of 33.61 out of $100(\mathrm{SD}=28.25)$. More than half of the respondents experienced similar public health crisis $(60.32 \%)$. Most participants had learnt COVID-19-related knowledge $(95.40 \%)$, primarily from the Internet $(89.25 \%)$ and television $(65.48 \%)$. The mean self-rated level of knowledge was 73.33 out of $100(\mathrm{SD}=20.92)$. More than half of the respondents reported needs for further education on COVID-19 (56.17\%), especially on the progress of the pandemic $(46.28 \%)$, personal protective measures $(45.38 \%)$, diagnostic technology and treatments (43.53\%).

\section{Anxiety and depression levels}

The mean score of anxiety and depression was 4.35 $(\mathrm{SD}=2.97)$ and $4.38(\mathrm{SD}=3.19)$, respectively. Based on the results of HADS, the rate of people with anxiety and depression symptoms (scores $>7$ ) was $14.15 \%$ and $17.35 \%$, respectively. As summarised in table 1, people with different characteristics reported different anxiety and depression levels, for example, age, gender, political party memberships, education levels, working status and conditions, living conditions, contact history, quarantines, wearing masks when going out, distance to the nearest COVID-19 patient and hospital, with COVID-19 patient(s) in the family/community, adoption of personal precautions, self-rated knowledge about COVID-19, selfperceived risks of getting infected, self-perceived health condition, needs of further COVID-19-related education and satisfaction to current precautions.

\section{Multiple linear regression analysis of anxiety and depression}

Multiple linear regression analysis (tables 2 and 3) revealed the following common risk factors for higher anxiety and depression levels: without political party membership, with contact history of COVID-19, going out or gathering, wearing masks, taking Chinese medicine herbs, being unsatisfied with current precautions, perceiving higher risks of infection, lower levels of knowledge and poorer health status. Furthermore, those who were female, married, lived alone and wore mask were more anxious; whereas people who were younger, experienced public health crisis, did not take precautions (regular work-rest, exercise) had higher depression level. Figure 1 shows the scores of anxiety and depression among different groups of participants.

\section{DISCUSSION}

During the rapid progressing stage of COVID-19 pandemic in China, one-seventh people presented anxiety symptoms, whereas one-sixth reported depressive symptoms. Females, those without political party membership, with contact history of COVID-19, going out or gathering, taking Chinese medicine herbs, being unsatisfied with current precautions, perceiving higher susceptibility, lower knowledge and poorer health status, reported higher anxiety and depression levels.

The current study was a nationwide online investigation with a large sample size and using validated study instrument. The participants perceived a high level of health (84.13 out of 100) and a low level of risk for infection $(33.61 \%)$, which were similar to previous findings. ${ }^{31} 32$ Consistent with other online investigations, females and younger people were more likely to respond. ${ }^{17-19} 3132$ Although $95.40 \%$ of the respondents had learnt related knowledge about COVID-19, their self-rated knowledge level was 73.33 out of 100 , indicating the need for further education on COVID-19. Consistent with previous studies during the pandemic, mass media including the Internet $(89.25 \%)$ and television $(65.48 \%)$ were the main resources of knowledge. ${ }^{33}$ The current findings indicated the needs and importance of equipping all levels of populations with COVID-19-related information by online or mass media delivered training/education. ${ }^{33-35}$ This study also reported a high adoption rate of precautionary measures. Although the majority of the respondents $(94.57 \%)$ did not contact with suspected or diagnosed COVID-19 cases, they strictly followed the precautions recommended by the NHC, such as wearing masks, not going our or gathering, washing hands and frequent ventilation. More than half of the respondents $(60.32 \%)$ had experienced SARS, which may explain their higher confidence about the effectiveness of these precautionary measures than those without such experience. Compared with other countries, China was seriously affected by SARS, and this experience may have contributed to the public's adherence to the recommended precaution measures, such as wearing masks. ${ }^{36}$

The $14.15 \%$ and $17.35 \%$ prevalence of anxiety and depression symptoms in the current study were similar to that in a previous one conducted at the peak of the COVID-19 outbreak in China $(13.5 \%$ for anxiety and $17.2 \%$ for depression). ${ }^{37}$ The rapidly increasing number of infected cases and deaths, national quarantine policy, fear of infection and uncertainty imposed psychological distress on the public. ${ }^{8}$ Another study in the initial stage of COVID-19 reported a similar prevalence of depression 


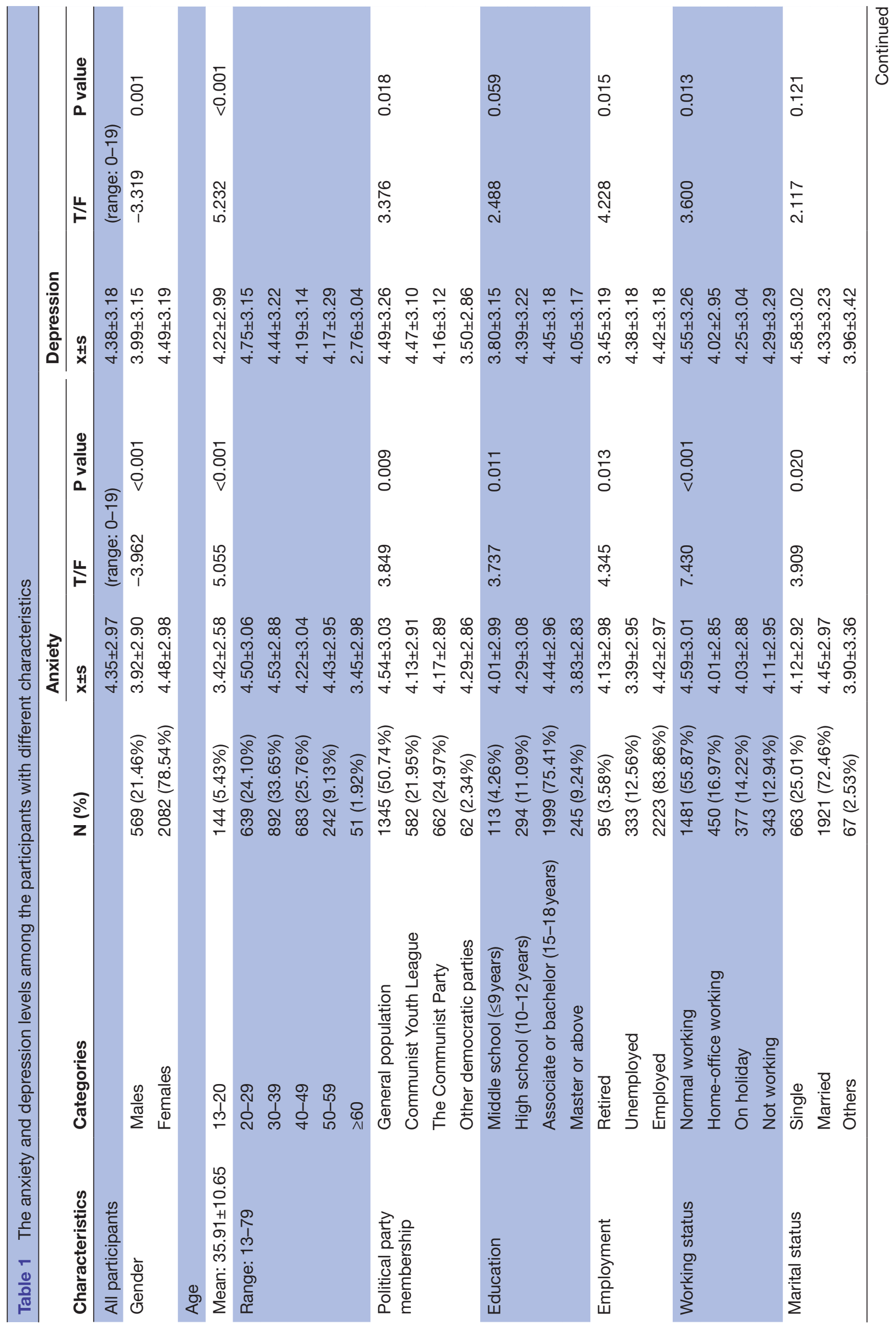




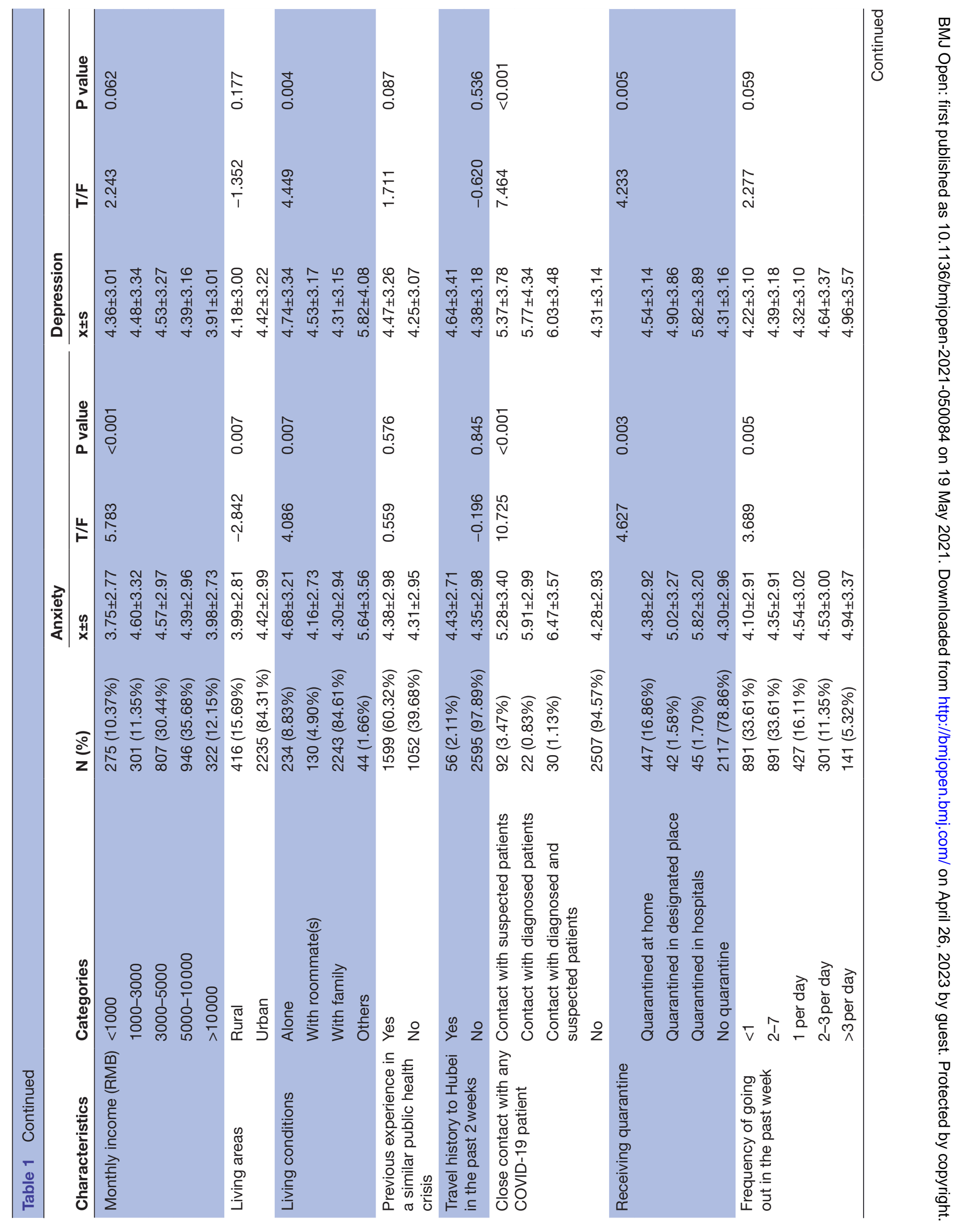




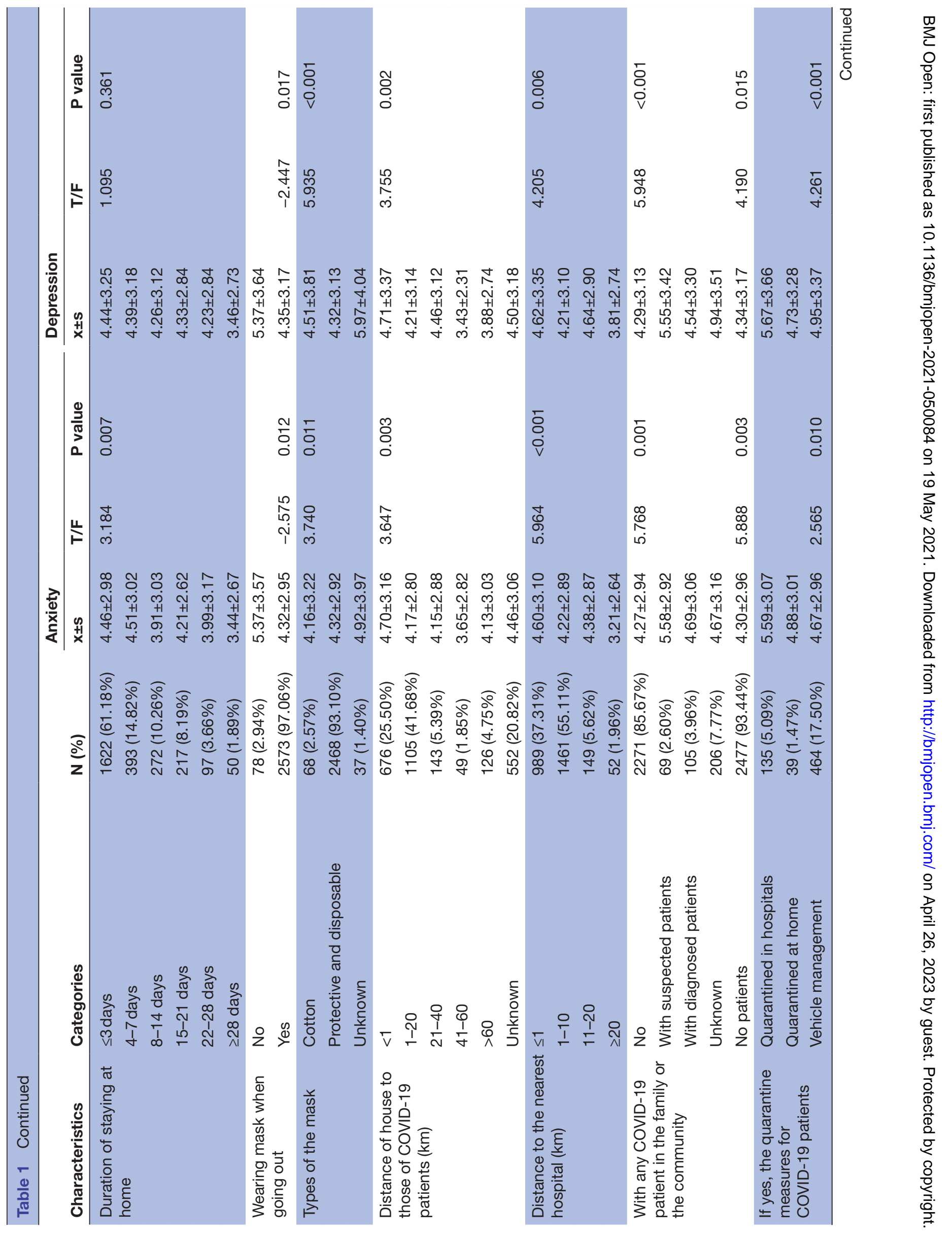




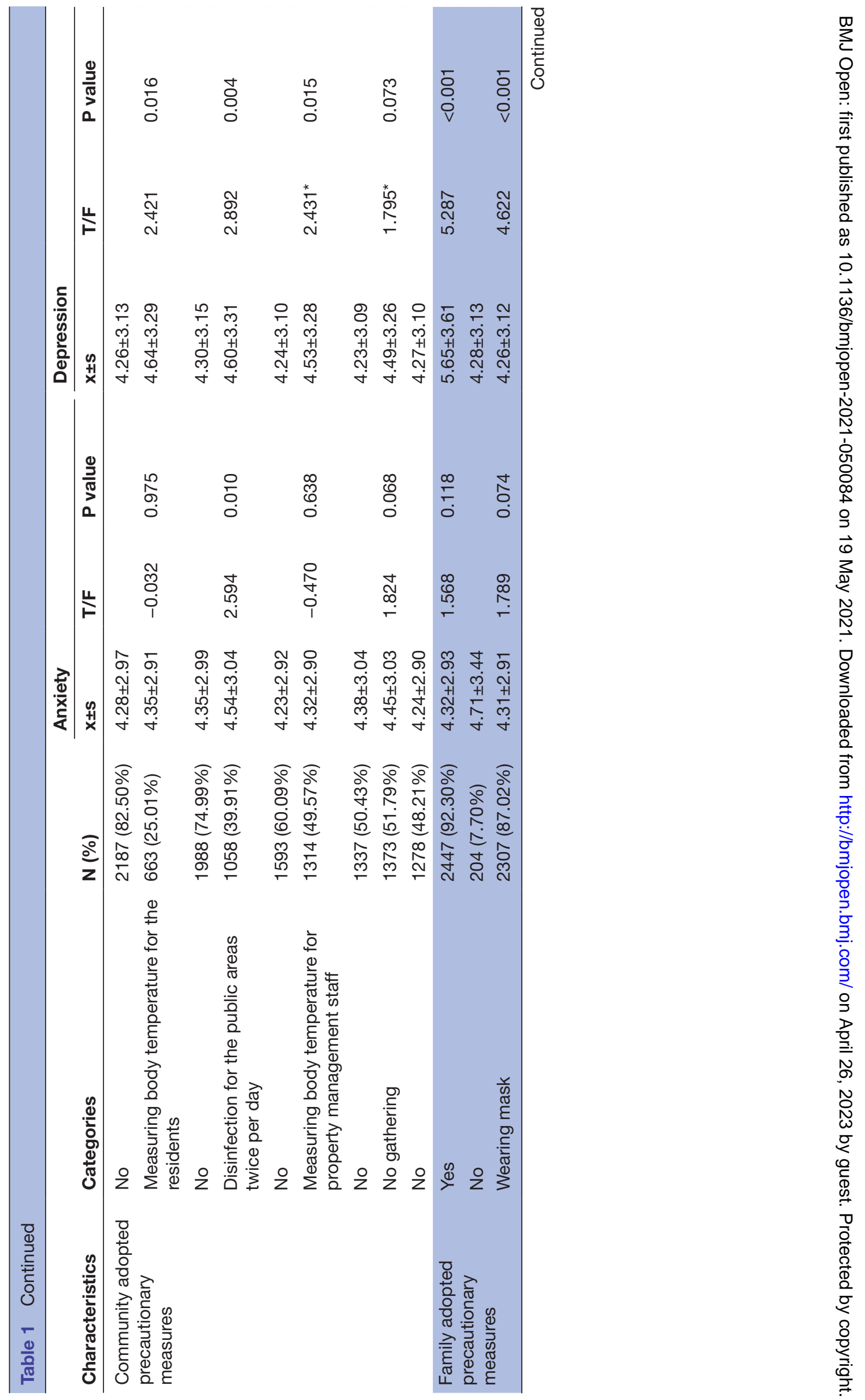




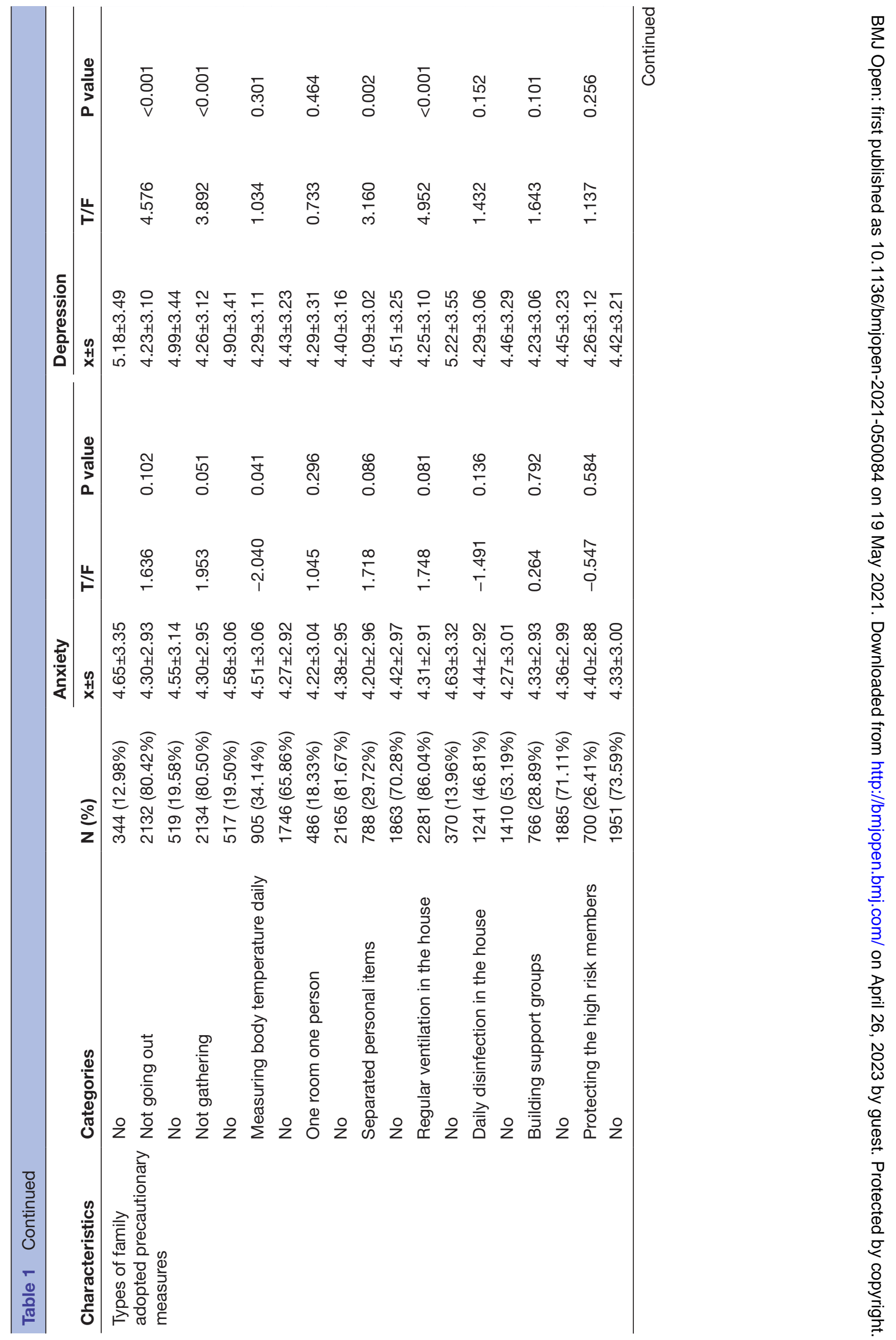




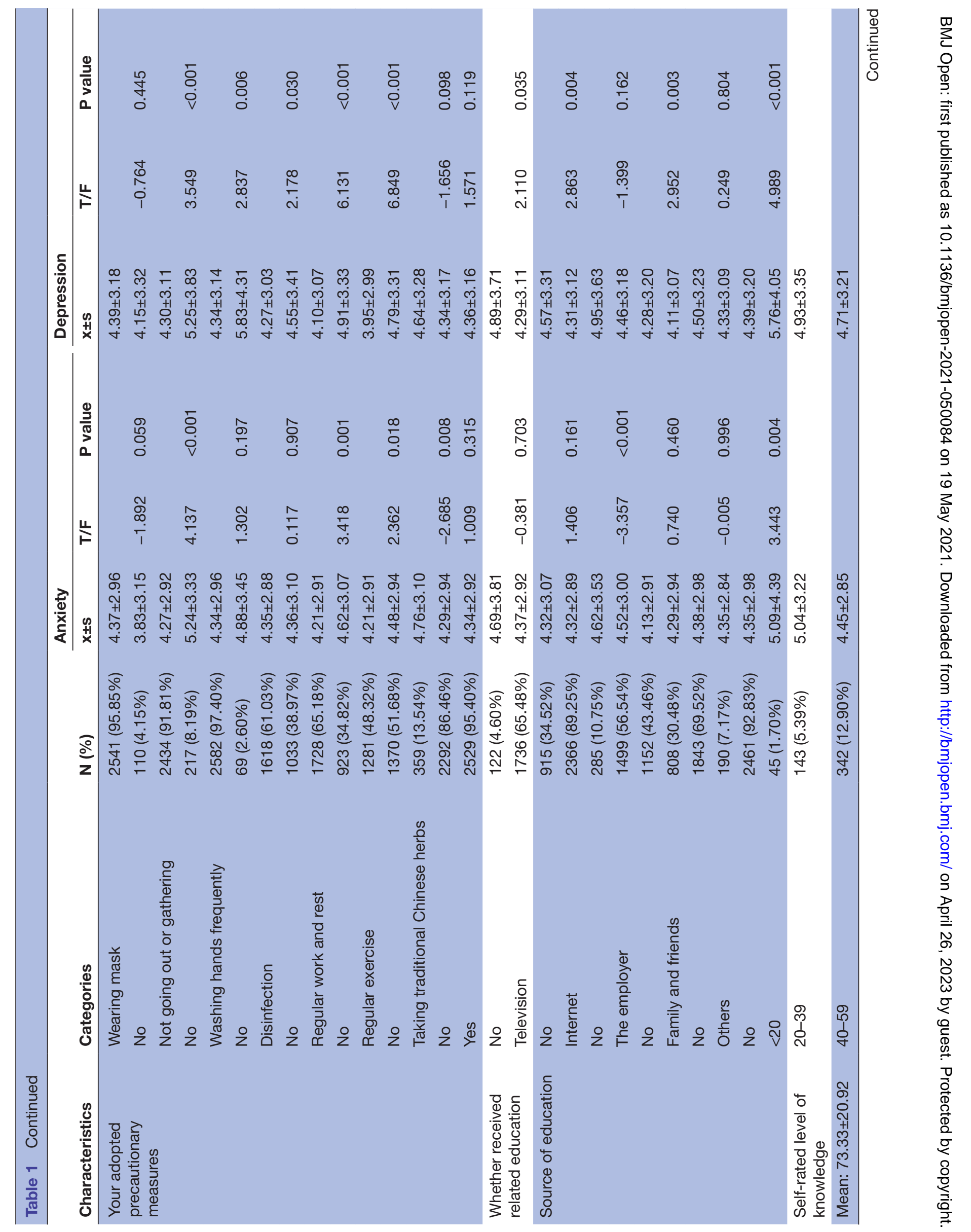




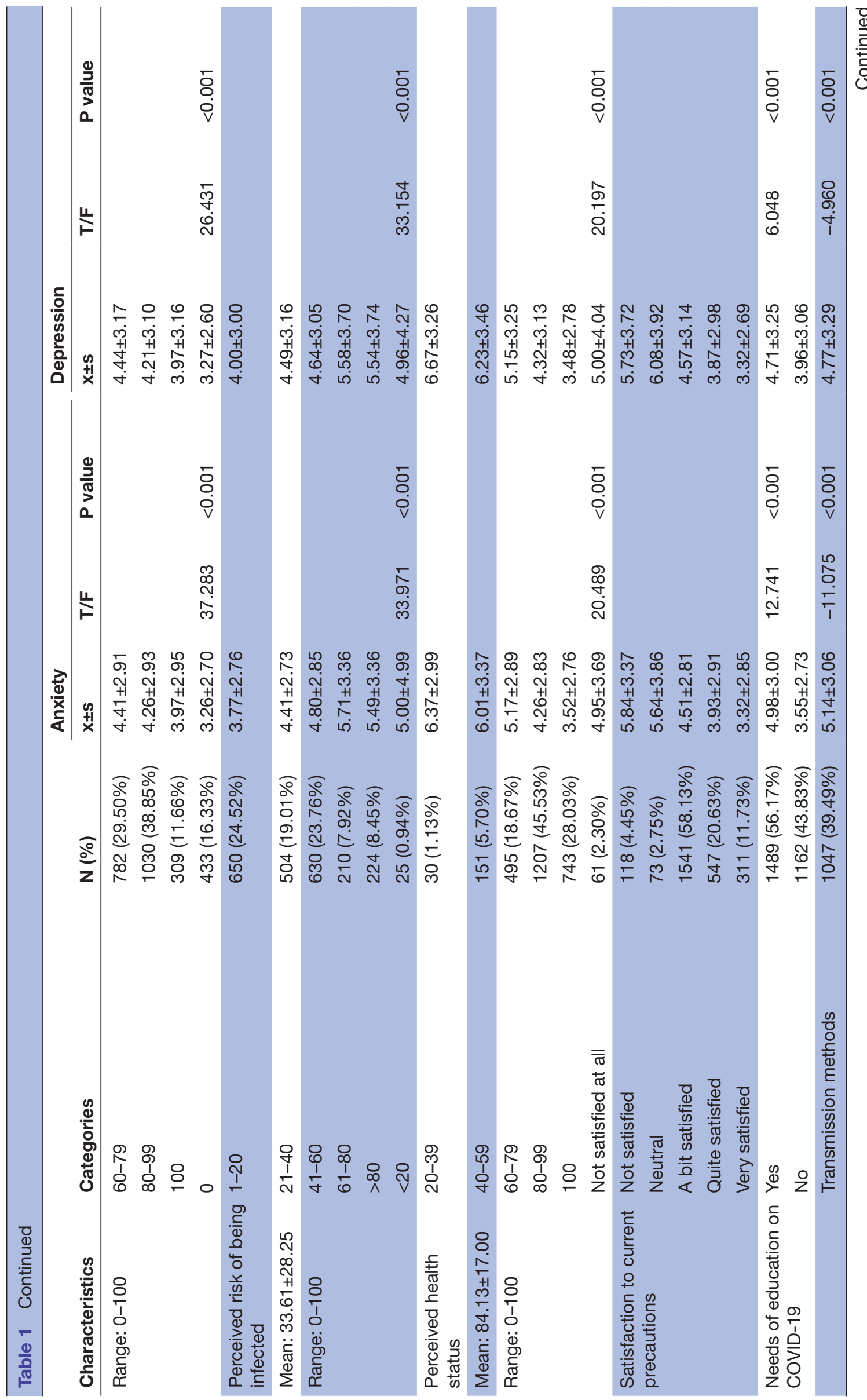




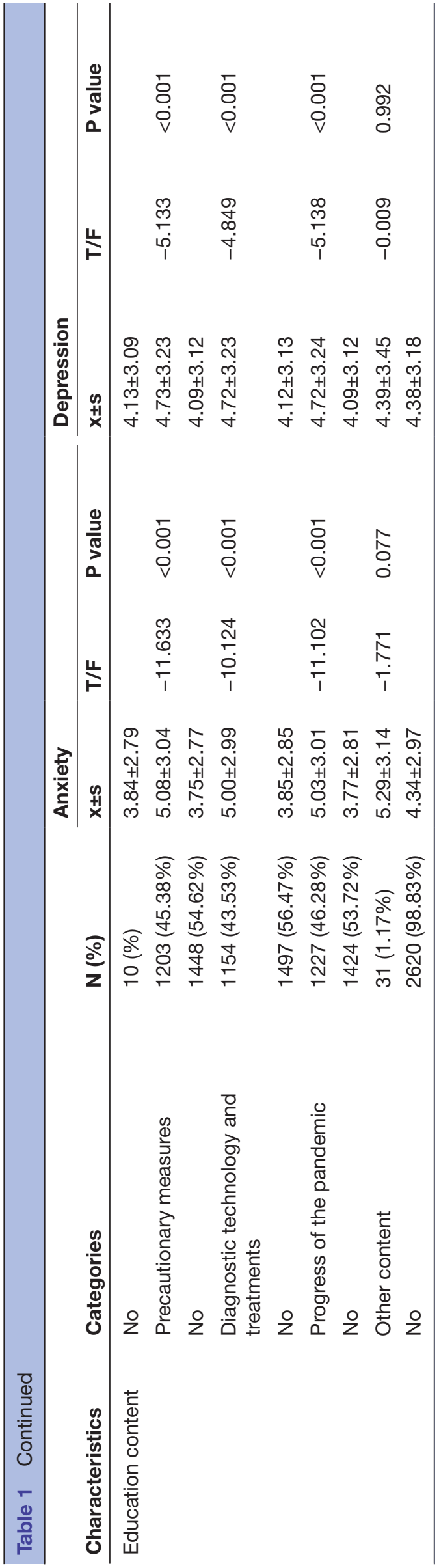

$(16.5 \%)$ and a higher prevalence of anxiety $(28.8 \%),{ }^{19}$ which may be associated with the uncertainty, lack of knowledge and lack of preparedness in that period. Moreover, the initial-stage study recruited more students, who were anxious about the pandemic and their academic progress. ${ }^{19}$ The prevalence of anxiety and depression in the public indicated the urgent needs of psychological support during an infectious disease epidemic. Online psychological support can be used, such as the Internet or digital cognitive behavioural therapy, which has the advantages of efficacy, cost-effectiveness and avoidance of personal contacts. ${ }^{38} 39$

\section{Factors associated with anxiety and depression levels}

The multiple linear regression model identified common factors associated with the anxiety and depression levels of the public. People who were not political party members, with close contact to COVID-19 patients, not following personal precautions of going out or wearing mask, taking traditional Chinese herbs, unsatisfied with current precautions, perceiving higher risks of being infected, perceiving lower level of knowledge and poorer health condition had higher anxiety and depression levels. Similar to previous findings, contact history, health status and perceived susceptibility are important predictors of psychological health. ${ }^{141722} 3132$ The worries about acquiring infection after close contact with patients induce higher anxiety and depression levels. ${ }^{17} 20$ Once the public perceive poor health and increased risks of infection, they become overwhelmed, anxious and depressed. ${ }^{10} 1415171922$ Satisfaction with the adequacy of government's preparedness would establish people's confidence to overcome this pandemic and alleviate the anxiety and depression. ${ }^{16} 19213132$ Lack of knowledge may trigger anxiety owing to fear of the unknown. ${ }^{10} 16172231$ Higher levels of self-rated knowledge facilitate people to take more reasonable responses. During the study period, the number of newly diagnosed COVID-19 patients reached the peak on 12 February 2020. ${ }^{5}$ Under such great changes in this pandemic, some people may feel fearful, hopeless or even panicky. ${ }^{13}$ Therefore, to reduce the anxiety and depression symptoms among the general population, the government should provide education (eg, the transmission methods) and promptly publish accurate progress (eg, medical supplies, case numbers and progress in treatment) through mass media. ${ }^{35}$

The findings also indicated Chinese specific social and cultural impacts on the psychological health of the general population. First, political party members presented lower anxiety and depression levels with the belongingness of political beliefs. A previous investigation also reported that nurses who were members of the Communist Party of China were more willing to join the aid team in Hubei. ${ }^{40}$ Another interesting finding was that people who took the individual precautions of wearing masks and use traditional Chinese herbs had higher anxiety and depression levels. This finding differed from another study that concluded face-masks use may 
Table 2 The multiple linear regression for the level of anxiety among the general population

\begin{tabular}{|c|c|c|c|c|c|}
\hline \multirow[b]{2}{*}{ Independent variables } & \multirow[b]{2}{*}{ B } & \multicolumn{2}{|l|}{$95 \% \mathrm{Cl}$} & \multirow[b]{2}{*}{ SE } & \multirow[b]{2}{*}{$P$ value } \\
\hline & & Lower & Upper & & \\
\hline Constant & 6.491 & 5.215 & 7.767 & 0.651 & $<0.001 \dagger$ \\
\hline Gender & 0.273 & 0.025 & 0.522 & 0.127 & $0.031^{*}$ \\
\hline Marital status & 0.467 & 0.236 & 0.699 & 0.118 & $<0.001 \dagger$ \\
\hline Political party membership & -0.147 & -0.259 & -0.034 & 0.058 & $0.011^{*}$ \\
\hline Living conditions & -0.392 & -0.728 & -0.055 & 0.172 & $0.022^{*}$ \\
\hline Contact history & 0.765 & 0.312 & 1.217 & 0.061 & $0.001 \dagger$ \\
\hline Wearing mask when going out & 0.825 & 0.214 & 1.436 & 0.312 & $0.008 \dagger$ \\
\hline Individuals precautionary measures_wearing mask & 0.686 & 0.167 & 1.206 & 0.265 & $0.010^{*}$ \\
\hline Individuals precautionary measures_not going out or gathering & -0.786 & -1.154 & -0.417 & 0.188 & $<0.001 \dagger$ \\
\hline Individuals precautionary measures_taking Chinese herbs & 0.483 & 0.190 & 0.777 & 0.150 & $0.001 \dagger$ \\
\hline Satisfaction to current precautions & -0.790 & -1.194 & -0.386 & 0.206 & $<0.001 \dagger$ \\
\hline Perceived risk of getting infected & 0.022 & 0.018 & 0.026 & 0.002 & $<0.001 \dagger$ \\
\hline Perceived health condition & -0.029 & -0.035 & -0.022 & 0.003 & $<0.001 \dagger$ \\
\hline Self-rated level of knowledge & -0.012 & -0.017 & -0.007 & 0.003 & $<0.001 \dagger$ \\
\hline
\end{tabular}

Adjusted $R^{2}=0.149, p<0.001$.

Dependent variable: the score of anxiety subscale. Independent variables: age, gender, education, political party membership employment status, marital status, monthly income, living areas and conditions, previous experience in public health crisis, travel history to Hubei, contact history with the patients, whether receive quarantine, frequency of going out, duration of staying at home, wearing mask when going out, whether with patient in the family or community, family and individual adopted precautions (seven variables), the needs of education, perceived health condition and risk of being infected, self-rated level of knowledge and satisfaction level to current precautions. ${ }^{*} \mathrm{P}<0.05$. $\dagger \mathrm{P}<0.01$

safeguard better mental health. ${ }^{36}$ That study compared the anxiety and depression levels between Poland (35.0\% wearing mask) and China (96.8\% wearing mask), and found that Polish respondents reported higher anxiety and depression levels than Chinese. ${ }^{36}$ In the current study, although the t-test indicated that those who wore masks when going out reported lower anxiety and depression levels, the multiple regression analyses provided more comprehensive models with consideration of other factors, such as the perceptions of susceptibility, personal health and knowledge. Due to the limitation of the study design, the current study can reveal only the significant association between adoption of these precautions and anxiety/depression, but cannot clarify their causal relationship. The reasons for higher levels of anxiety/depression among people who took Chinese herbs or wore masks could be explored in future studies.

Besides the common factors, the present study also identified that those who were female, married and living alone had a higher level of anxiety. Previous studies also reported females were more vulnerable to stress ${ }^{41}$ and more likely to present anxiety symptoms during the public health crisis. ${ }^{10} 141719$ Compared with the single ones, married people had to care about their spouse and children during the pandemic, leading to a higher level of anxiety. ${ }^{1720} 23$ Consistent with previous studies, those who lived alone under the quarantine policy were more likely to feel lonely and present anxiety symptoms, indicating the importance of social support for psychological health. ${ }^{121520} 243132$

The participants who were younger, with experience in public health crisis reported higher depression levels, whereas who took precautions in families, regularly rested and exercised had lower depression levels. This finding was consistent with a previous one that younger people may have more access to information about the pandemic, thereby inducing depression. Moreover, as the key working force in the society, younger people may be more worried about their career and economic loss caused by the pandemic, so they presented higher depression levels. ${ }^{12} 141720313237$ As previously discussed, the adoption of precautions either by family or individuals can help to reduce the depression symptoms. Previous studies have commonly reported exercise as an effective approach to relieve depression in various populations. ${ }^{37}$ Moreover, precautionary measures such as regular rest and exercise may increase the confidence of individuals about their health, thereby reducing depression. ${ }^{42}$ These findings indicated the importance of introducing precautions and healthy lifestyles during this pandemic.

The current study has some limitations. First, this online investigation employed snowball sampling, which may induce bias in respondents' characteristics. For example, the proportion of males and old participants was low, similar as reported in other online investigations. ${ }^{10} 193132$ Caution is needed when generalising the findings to other 
Table 3 The multiple linear regression for the level of depression among the general population

\begin{tabular}{lllllll} 
& & & $95 \% \mathbf{C l}$ & & & \\
Independent variables & & & Lower & Upper & SE & P value \\
\hline Constant & 9.307 & 8.132 & 10.481 & 0.599 & $<0.001 \dagger$ \\
\hline Age & -0.023 & -0.034 & -0.013 & 0.005 & $<0.001 \dagger$ \\
\hline Gender & 0.273 & 0.001 & 0.546 & 0.139 & 0.050 \\
\hline Political membership & -0.138 & -0.261 & -0.015 & 0.063 & $0.028^{*}$ \\
\hline Contact history & 0.581 & 0.086 & 1.076 & 0.252 & $0.021^{*}$ \\
\hline Previous experience in public health crisis & 0.412 & 0.182 & 0.642 & 0.117 & $<0.001 \dagger$ \\
\hline Whether family adopted precautionary measures & -1.138 & -1.557 & -0.719 & 0.214 & $<0.001 \dagger$ \\
\hline Individuals precautionary measures_not going out or gathering & -0.533 & -0.940 & -0.126 & 0.208 & $0.010^{*}$ \\
\hline Individuals precautionary measures_wearing mask & 0.591 & 0.035 & 1.147 & 0.283 & $0.037^{*}$ \\
\hline Individuals precautionary measures_regular work and rest & -0.326 & -0.585 & -0.068 & 0.132 & $0.013^{*}$ \\
\hline Individuals precautionary measures_regular exercise & -0.307 & -0.551 & -0.063 & 0.124 & $0.014^{*}$ \\
\hline Individuals precautionary measures_taking Chinese herbs & 0.428 & 0.107 & 0.749 & 0.164 & $0.009 \dagger$ \\
\hline Satisfaction to current precautions & -0.720 & -1.161 & -0.279 & 0.225 & $0.001 \dagger$ \\
\hline Perceived risk of getting infected & 0.018 & 0.014 & 0.022 & 0.002 & $<0.001 \dagger$ \\
\hline Perceived health condition & -0.031 & -0.038 & -0.024 & 0.003 & $<0.001 \dagger$ \\
\hline Self-rated level of knowledge & -0.010 & -0.015 & -0.004 & 0.003 & $0.001 \dagger$ \\
\hline
\end{tabular}

Adjusted $R^{2}=0.134, p<0.001$.

Dependent variable: the score of depression subscale. Independent variables: age, gender, education, political party membership employment status, marital status, monthly income, living areas and conditions, previous experience in public health crisis, travel history to Hubei, contact history with the patients, whether receive quarantine, frequency of going out, duration of staying at home, wearing mask when going out, whether with patient in the family or community, family and individual adopted precautions (seven variables), the needs of education, perceived health condition and risk of being infected, self-rated level of knowledge and satisfaction level to current precautions. ${ }^{*} \mathrm{P}<0.05$.

$\dagger \mathrm{P}<0.01$

populations. Future study could employ a random-based sampling to recruit a more representative sample. Second, this cross-sectional study was conducted during the rapid progressing stage in this epidemic. Longitudinal studies could be conducted to explore the dynamic changes in psychological health. Qualitative studies are also recommended to explore in-depth information about people's psychological responses and related factors. Moreover, the possible factors associated with anxiety and depression were explored based on literature review and measured by simple questions. For example, health condition and COVID-19 knowledge were measured only by single VAS items. Future studies could explore the influencing factors within the conceptual framework,
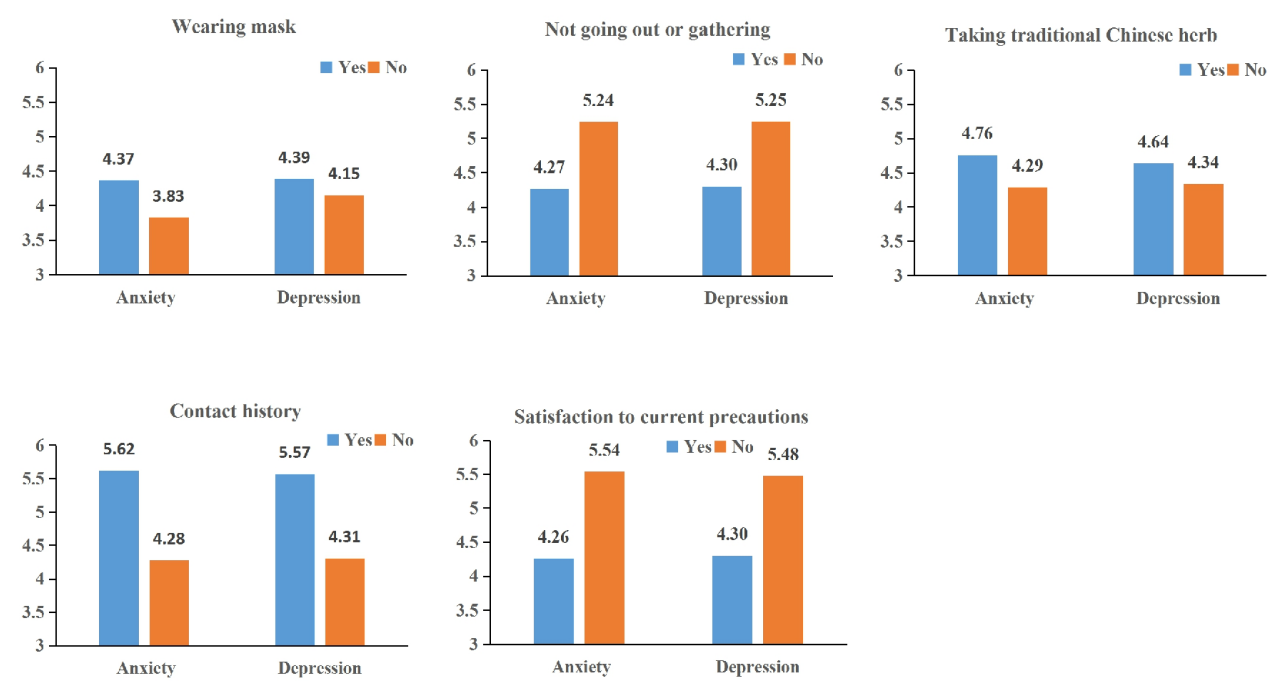

Figure 1 Comparison of anxiety and depression scores between different groups of participants. 
such as the Theory of Stress. Specific study instruments, such as a COVID-19 knowledge scale, should be employed to provide more detailed information. Qualitative studies could be designed to explore the reasons of higher levels of anxiety/depression among people who took Chinese herbs and wore masks.

The prevalence of anxiety (one-seventh) and depression (one-sixth) in the public indicated the importance and need of psychological support during a public health crisis. Individuals should follow the NHC guidelines on precautions and healthy advice to reduce the possibility of infection and improve psychological health. The government should provide extensive information on coronavirus-related knowledge, transmission methods, precautionary measures and progress of the pandemic through mass media. Online psychological support could be provided for the high risk group of people to combat the adverse psychological impacts of COVID-19.

\section{CONCLUSION}

During the rapid progressing stage of the COVID-19 pandemic in China, one-seventh and one-sixth respondents presented anxiety and depression symptoms, respectively. The risk factors for anxiety and depression included the following: without political party membership, with contact history of COVID-19, going out or gathering, wearing masks, taking Chinese medicine herbs, being unsatisfied with current precautions, perceiving higher susceptibility, lower knowledge and poorer health status. Extensive information and online psychological support should be provided to the general population, especially the high-risk groups for psychological disorders during a public health crisis.

\author{
Author affiliations \\ ${ }^{1}$ Nursing Department, Hunan Provincial People's Hospital, Changsha, China \\ ${ }^{2}$ The Nethersole School of Nursing, Chinese University of Hong Kong, New \\ Territories, Hong Kong \\ ${ }^{3}$ Teaching and Research Section of Clinical Nursing, Xiangya Hospital Central South \\ University, Changsha, Hunan, China \\ ${ }^{4}$ School of Basic Medicine, North Sichuan Medical University, Nanchong, Sichuan, \\ China \\ ${ }^{5}$ School of Nursing, Shenzhen University, Shenzhen, China
}

Acknowledgements The authors would like to thank all the respondents of this investigation for their participation.

Contributors ZS and YQ contributed to study design, data collection and analysis, resources, manuscript writing. SYC contributed to study design, supervision and validation, writing, review and editing of the manuscript. YL, YT, XL and WH performed data collection and analysis, project administration and manuscript writing. QW contributed to study design, data analysis, writing, review and editing of the manuscript. All authors read and approved the final manuscript. ZS and YQ contributed equally for this manuscript.

Funding The study was supported by the grants from the Health Commission of Guangdong Province (Fund no. A2019067) and Top Ranking Projects of Shenzhen University (no. 86000000210 and no. 860000002110118 ). The funding source had no influence on the study design, procedure, data analysis or interpretations of the findings.

Competing interests None declared.

Patient consent for publication Not required.
Ethics approval The study was approved by the Ethical Committee of Hunan Province People Hospital (No. 2020007). Electronic informed consent was obtained from each participant.

Provenance and peer review Not commissioned; externally peer reviewed.

Data availability statement Data are available upon reasonable request. The data that support the findings in this study are available from the corresponding author upon reasonable request.

Supplemental material This content has been supplied by the author(s). It has not been vetted by BMJ Publishing Group Limited (BMJ) and may not have been peer-reviewed. Any opinions or recommendations discussed are solely those of the author(s) and are not endorsed by BMJ. BMJ disclaims all liability and responsibility arising from any reliance placed on the content. Where the content includes any translated material, BMJ does not warrant the accuracy and reliability of the translations (including but not limited to local regulations, clinical guidelines, terminology, drug names and drug dosages), and is not responsible for any error and/or omissions arising from translation and adaptation or otherwise.

Open access This is an open access article distributed in accordance with the Creative Commons Attribution Non Commercial (CC BY-NC 4.0) license, which permits others to distribute, remix, adapt, build upon this work non-commercially, and license their derivative works on different terms, provided the original work is properly cited, appropriate credit is given, any changes made indicated, and the use is non-commercial. See: http://creativecommons.org/licenses/by-nc/4.0/.

ORCID iDs

Zeya Shi http://orcid.org/0000-0002-9291-6205

Qun Wang http://orcid.org/0000-0001-7594-8312

\section{REFERENCES}

1 Chen J. Pathogenicity and transmissibility of 2019-nCoV-A quick overview and comparison with other emerging viruses. Microbes Infect 2020;22:69-71.

2 World Health Organization. Novel coronavirus (2019-nCoV) situation Report-11 (data as reported by 31 January 2020. Geneva: World Health Organization, 2020. https://www.who.int/docs/default-source/ coronaviruse/situation-reports/20200131-sitrep-11-ncov.pdf?sfvrsn= de7c0f7_4

3 National Health Commission of the People's Republic of China. Updates on COVID-19 by 9 February (24:00), 2020. Available: http:// www.nhc.gov.cn/xcs/yqtb/202002/167a0e01b2d24274b03b2ca9 61107929.shtml

4 National Health Commission of the People's Republic of China. Updates on COVID-19 by 19 February (24:00), 2020. Available: http://www.nhc.gov.cn/xcs/yqtb/202002/4dcfcb9b74ea4a408fc1 d56d4db61f93.shtml

5 National Health Commission of the People's Republic of China. Updates on COVID-19 by 12 February (24:00), 2020, 2020c. Available: http://www.nhc.gov.cn/xcs/yqtb/202002/26fb16805f02 4382bff1de80c918368f.shtml

6 World Health Organization. WHO coronavirus disease (COVID-19) dashboard. Geneva: World Health Organization, 2021. https:// covid19.who.int/

7 National Health Commission of the People's Republic of China. Press conference on the novel coronavirus 2019 on 20 Feb 2020, 2020. Available: http://www.nhc.gov.cn/xcs/s3574/202002/526411b8aa61 43a487a6827aaa2f40ac.shtml

8 Van Bortel T, Basnayake A, Wurie F, et al. Psychosocial effects of an Ebola outbreak at individual, community and international levels. Bull World Health Organ 2016;94:210-4.

$9 \mathrm{CH} \mathrm{K}$, Yen CF, Yen JY. Psychosocial impact among the public of the severe acute respiratory syndrome epidemic in Taiwan. Psychiatry and Clinical Neurosciences 2016;60:397-403.

10 Hawryluck L, Gold WL, Robinson S, et al. Sars control and psychological effects of quarantine, Toronto, Canada. Emerg Infect Dis 2004;10:1206-12.

11 Mak IWC, Chu CM, Pan PC, IWC M, MGC Y, et al. Long-Term psychiatric morbidities among SARS survivors. Gen Hosp Psychiatry 2009;31:318-26.

12 Jeong H, Yim HW, Song Y-J, et al. Mental health status of people isolated due to middle East respiratory syndrome. Epidemiol Health 2016;38:e2016048.

13 National Health Commission of the People's Republic of China. Intervention guidelines for psychological crisis during COVID-19 epidemic, 2020e. Available: http://www.nhc.gov.cn/jkj/s3577/ 202001/6adc08b966594253b2b791be5c3b9467.shtml 
14 Leung GM, Ho L-M, Chan SKK, et al. Longitudinal assessment of community psychobehavioral responses during and after the 2003 outbreak of severe acute respiratory syndrome in Hong Kong. Clin Infect Dis 2005;40:1713-20.

15 AINajjar NS, Attar LM, Farahat FM, et al. Psychobehavioural responses to the 2014 middle East respiratory syndromenovel corona virus (MERS CoV) among adults in two Shopping malls in Jeddah, Western Saudi Arabia. East Mediterr Health $J$ 2017;22:817-23.

16 Deurenberg-Yap M, Foo LL, Low YY, et al. The Singaporean response to the SARS outbreak: knowledge sufficiency versus public trust. Health Promot Int 2005;20:320-6.

17 Salari N, Hosseinian-Far A, Jalali R, et al. Prevalence of stress, anxiety, depression among the general population during the COVID-19 pandemic: a systematic review and meta-analysis. Global Health 2020;16:57.

18 da Silva ML, Rocha RSB, Buheji M, et al. A systematic review of the prevalence of anxiety symptoms during coronavirus epidemics. $J$ Health Psychol 2021;26:115-25.

19 Wang C, Pan R, Wan X, et al. Immediate psychological responses and associated factors during the initial stage of the 2019 coronavirus disease (COVID-19) epidemic among the general population in China. Int J Environ Res Public Health 2020;17:1729.

20 Wang S, Zhang Y, Ding W, et al. Psychological distress and sleep problems when people are under interpersonal isolation during an epidemic: a nationwide multicenter cross-sectional study. European Psychiatry 2020;63:e77:1-8

21 Dai XR, Liu T, Liu YW. Psychological status and influencing factors of high school students in Chengdu during the outbreaks of COVID-19. Modern Preventive Medicine 2020;47:3911-4.

22 Huang $Y$, Wang $Y$, Zeng $L$, et al. Prevalence and correlation of anxiety, insomnia and somatic symptoms in a Chinese population during the COVID-19 epidemic. Front. Psychiatry 2020;11:1-8.

23 Zhang $Y$, Wang S, Ding W, et al. Status and influential factors of anxiety depression and insomnia symptoms in the work resumption period of COVID-19 epidemic: a multicenter cross-sectional study. $J$ Psychosom Res 2020;138:110253-8.

24 Deng ET, Liu YL, Cheng SY. Investigation on psychological status of the people under coronavirus disease. Journal of North China University of Science and Technology 2020;22:482-8.

25 Barczak P, Kane N, Andrews S, et al. Patterns of psychiatric morbidity in a genito-urinary clinic. A validation of the hospita anxiety depression scale (had). Br J Psychiatry 1988;152:698-700.

26 Wang W, Chair SY, Thompson DR, et al. A psychometric evaluation of the Chinese version of the hospital anxiety and depression scale in patients with coronary heart disease. J Clin Nurs 2009;18:2436-43.

27 Sun JW, Han Y, Bai HY. Psychological stress reactions of occupational exposure to blood-borne infectious pathogens among medical staff: a longitudinal study. Chinese Mental Health Journal 2017;31:190-4.

28 Su Q, Liu YP, Cheng YF. The study on reliability and validity of hospital anxiety and depression scale among physical examination people. Sichuan Medical 2012;33:174-6.

29 Tabachnick BG. Multiple Regression. In: Tabachnick BG, Fidel LS, eds. Using multivariate statistics. 7th ed. Boston: Pearson Education, 2019: 104-9.

30 Zhang WT, Dong W. Chapter 6: multiple linear regression model. In: Advanced textbook for statistical analyses by SPSS. 3rd. Beijing: Higher Education Press, 2018: 101-23.

31 Wang $C$, Tee M, Roy AE, et al. The impact of COVID-19 pandemic on physical and mental health of Asians: a study of seven middleincome countries in Asia. PLoS One 2021;16:e0246824.

32 Tee ML, Tee CA, Anlacan JP, et al. Psychological impact of COVID-19 pandemic in the Philippines. J Affect Disord 2020;277:379-91.

33 Tran BX, Dang AK, Thai PK, et al. Coverage of health information by different sources in communities: implication for COVID-19 epidemic response. Int J Environ Res Public Health 2020;17:3577.

34 Le HT, Mai HT, Pham HQ, et al. Feasibility of Intersectoral collaboration in epidemic preparedness and response at Grassroots levels in the threat of COVID-19 pandemic in Vietnam. Front Public Health 2020;8:589437.

35 Tran BX, Phan HT, Nguyen TPT, et al. Reaching further by village health collaborators: the informal health Taskforce of Vietnam for COVID-19 responses. J Glob Health 2020;10:010354.

36 Wang C, Chudzicka-Czupała A, Grabowski D, et al. The association between physical and mental health and face mask use during the COVID-19 pandemic: a comparison of two countries with different views and practices. Frontiers in Psychiatry 2020;11:569981.

37 Huang J, Liu F, Teng Z, et al. Public behavior change, perceptions, depression, and anxiety in relation to the COVID-19 outbreak. Open Forum Infect Dis 2020;7:1-8.

38 Soh HL, Ho RC, Ho CS, et al. Efficacy of digital cognitive behavioural therapy for insomnia: a meta-analysis of randomised controlled trials. Sleep Med 2020;75:315-25.

39 Zhang MWB, Ho RCM. Moodle: the cost effective solution for Internet cognitive behavioral therapy (I-CBT) interventions. Technol Health Care 2017;25:163-5.

40 Gan XQ, Shi ZY, Chair SY. Willingness of Chinese nurses to practice in Hubei combating the coronavirus disease 2019 epidemic: a crosssectional study. J Adv Nurs 2020;76:2137-50.

41 Lim GY, Tam WW, Lu Y, et al. Prevalence of depression in the community from 30 countries between 1994 and 2014. Sci Rep 2018;8:2861.

42 Wang Q, Chair SY, Wong EM-L. The effects of a lifestyle intervention program on physical outcomes, depression, and quality of life in adults with metabolic syndrome: a randomized clinical trial. Int $J$ Cardiol 2017;230:461-7 